\title{
The Effect of Interval Training on Oxidative Stress Indices Among Women in Preterm Labor Underwent Coronary Artery Bypass Graft
}

\author{
Khosrow Hashemzadeh $^{\circledR}$, Marjan Dehdilani², Mehdi Khanbabayi Gol ${ }^{3^{*}(\mathbb{D}}$
}

\begin{abstract}
Objectives: Oxidative stress indices are one of the factors that cause and exacerbate cardiovascular diseases in postpartum women, which can be changed with interval training. Therefore, the current study aimed at investigating the effect of interval training on oxidative stress indices among women in preterm labor undergoing the coronary artery bypass graft (CABG).

Materials and Methods: This randomized clinical trial was conducted on 21 participants (the sample size was determined based on a similar study) from December 2018 to May 2019 at Shahid Madani hospital of Tabriz, Iran. After the random allocation of subjects to the training $(\mathrm{n}=11)$ and control $(\mathrm{n}=10)$ groups, demographic information was collected and their physical activity level was assessed using the Kaiser physical activity survey before and after the intervention. In addition, the levels of oxidative stress indices were measured 24 hours before and after the intervention using the blood sample. The participants completed an eight-week invention including 24 one-hour sessions according to the available protocol. Finally, data were analyzed by means of statistical tests such as the Shapiro-Wilk, Leven, and Student's t-test at a significant level of $<0.05$.

Results: The effect of the aerobic exercise program on body weight loss, as well as body mass index (BMI) and body fat percentage changes was significant in the training group $(\mathrm{P}=0.011, \mathrm{P}=0.004$, and $\mathrm{P}=0.001$, respectively). Based on the results, the serum and urine levels of 8-hydroxy-2-deoxyguanosine reduced significantly after the completion of the intervention $(\mathrm{P}=0.002$ and $\mathrm{P}=0.001$, respectively) in the training group.

Conclusions: In general, aerobic exercise after CABG decreased the fat percentile, BMI, and oxidative stress indices in the subjects. Thus, these trainings could reduce oxidative stress indices by increasing tissue metabolism and oxygen consumption.

Keywords: Oxidative Stress, Labor, CABG
\end{abstract}

\section{Introduction}

Numerous studies showed that oxidative stress can be considered as a leading factor in the pathogenesis of some chronic non-communicable diseases such as diabetes, cancer, chronic kidney disease, and cardiovascular disease, as well as the incidence of some processes including aging $(1,2)$. Oxidative stress is a condition in which the balance between the production of free radicals and their neutralization by antioxidants is disturbed, namely, the condition that leads to the accumulation of free radicals and their production inside the cells (3), consequently exacerbating the severity of the disease and its complications (4). In addition, oxidative stress is recognized as a pathogen in most diseases $(5,6)$, and important sources of oxidative stress in the body are environmental contaminants, rays, and chemical compounds such as anticancer drugs, tobacco, and alcohol (7).

It is shown that oxidative stress can cause premature labor in women. In other words, women in premature labor have high levels of oxidative stress (8). On the other hand, oxidative stress leads to postpartum complications in women (9).

According to previous research, there is a close relationship between the production of reactive oxygen species (ROS) and the antioxidant system analysis (10). ROS is one of the factors affecting preterm labor which, according to (11), can cause preterm labor by affecting the antioxidant system. Likewise, structural changes in DNA as a result of exposure to ROS have attracted the attention of some researchers, and such changes play an important role in the development of aging, atherosclerosis, and diabetes (12).

Today, many interventions relying on medication, improvement of dietary habits, dietary supplements, and regular exercise are used to control oxidative conditions in the heart and prevent cardiovascular diseases (13), and their impact on oxidative stress indices is also confirmed in some studies while rejected in some other (14-16).

The increase in age worsens the condition of

Received 4 June 2019, Accepted 9 September 2019, Available online 5 October 2019

${ }^{1}$ Department of Heart Surgery, Tabriz University of Medical Sciences, Tabriz, Iran. ${ }^{2}$ Department of Anesthesiology, Tabriz University of Medical Sciences Tabriz, Iran. ${ }^{3}$ Responsible for the Nursing Research Committee of Imam Reza Hospital, Nursing and Midwifery Faculty, Tabriz University of Medical Sciences, Tabriz, Iran.

*Corresponding Author: Mehdi Khanbabayi, Tel:+989141855143, Email: Mkhanbabayi@yahoo.com 
Key messages

- Oxidative stress indices are one of the factors that cause cardiovascular diseases in postpartum women

- Aerobic exercise affects oxidative stress indices.

- Aerobic exercise leads to positive effects on oxidative stress indices in women with preterm delivery.

cardiovascular disease (17), and women who have had premature delivery also higher levels of oxidative stress as well. On the other hand, coronary artery bypass graft (CABG) is the treatment of choice for narrowed or blocked coronary arteries, and it is expected that the health status dramatically improves after CABG in the affected patients while many of such patients continue to experience significant problems after CABG. Therefore, prevention from post-CABG complications in the affected patients is part of the treatment process. Given that very few studies are conducted on the effects of interval training on the reduction of oxidative stress after premature delivery (one of the causing and exacerbating factors for heart disease), and no study thus far has investigated the effects of interval training on post-CABG oxidative stress in women with preterm delivery, the present study aimed at evaluating the effect of interval training on oxidative stress indices in women with preterm delivery undergoing CABG surgery.

\section{Materials and Methods}

The current parallel-group randomized, controlled trial was conducted from 22 December 2018 to 22 May 2019 at Shahid Madani Hospital in Tabriz, Iran. The inclusion criteria were female gender, an experience of preterm delivery, permission for exercise (confirmed by the relevant physician), the age range of 30 to 40 years, a body mass index (BMI) $\geq 19$ and $\leq 30 \mathrm{~kg} / \mathrm{m}^{2}$, and 6 months elapse of CABG surgery. On the other hand, the exclusion criteria included smoking and having regular training in the past three months and irregular training during the intervention.

The sample size was determined $21 \quad(n=11$ and $\mathrm{n}=10$ in the training and control groups, respectively). Participants were entered into the study using convenient and purposive sampling methods and were randomly divided into two groups using a random numbers table. In addition, random assignment to groups was performed by a statistical advisor.

After providing eligible participants with explanations about the study by the researcher's assistant, the participants were asked to complete the forms at the hospital. The forms had two parts. The first section included demographic information (i.e., age, height, weight, body fat mass, BMI, and the serum and urine levels of 8-hydroxy-2-deoxyguanosine) and the second one was the Kaiser physical activity survey the validity of which was 0.87 based on Cronbach's alpha (18).
Further, the body composition was analyzed as the height (measured to the nearest $5 \mathrm{~mm}$ using a statiometer, Seca, Germany), the hips and waist circumferences (measured to the nearest $5 \mathrm{~mm}$ using a tape), weight (by a digital weight scale to the nearest $100 \mathrm{~g}$ ), and body fat percentage (using a bioelectrical impedance device, inbody-720, South Korea).

Waist-to-hip ratio and BMI were measured. BMI was calculated by dividing weight in kilograms by height in meters squared. To measure the waist-to-hip ratio, the researcher first measured the waist circumference and then the hip circumference using a tape. Then, the waist-to-hip ratio was obtained by dividing the waist measurement by the hip measurement. All measurements were taken while the participants were prohibited from eating and drinking for four hours before the test, and their bladders, stomachs, and intestines were evacuated as far as possible.

In the current study, blood samples were collected 24 hours before and after the training session. Furthermore, sampling was performed in the laboratory from 8:00 to 10:00 AM, from the left ventricle of each subject in a sitting position and at rest by a skilled nurse who was blind to grouping. Moreover, the serum level of 8-hydroxy-2deoxyguanosine was measured by the enzyme-linked immunosorbent assay using Casa Bio kits (Japan).

The training program was an eight-week intervention including three 60-minute sessions per week. Additionally, morning exercises (9:30 to 11:00 AM) included general warm-ups for 10 minutes (i.e., walking, soft running, stretching exercises, and jugging) and aerobic exercises for $45-60$ minutes with an intensity of $50 \%-70 \%$ of the maximum heart rate. In addition, the sessions began from 30 minutes and then gradually increased to 45 minutes at the end of the intervention. Further, the intensity of the exercise was controlled by Pulsers ${ }^{\mathrm{TM}}$ (POLAR, Finland). At the end of each session, 10 minutes was dedicated to turning the body to its normal state and cooling-down (i.e., slow running, walking, and stretching exercises). After the completion of the intervention, body composition parameters were measured in a manner similar to those of pre-intervention measurements, and the data were collected accordingly. Furthermore, the intensity of exercise in addition to the heart rate was controlled by the Borg rating of perceived exertion scale (19). On the other hand, the control group had no activity during the study period and was inactive (had a normal lifestyle). Among the safety issues considered during the exercise, covering the gym floor with tatami mats to prevent damage to subjects seems noteworthy. The subjects were requested to have the right clothing and wear sports shoes at training sessions. Moreover, trainings were performed in the university gymnasium and supervised by the faculty members of the Physical Education Department, Tabriz University of Medical Sciences.

The study was carried out after obtaining approvals 
from the Ethics Committee of Tabriz University of Medical Sciences and the registration of the study in the Iranian Registry of Clinical Trials (No: IRCT20190325043107N2). After obtaining the required permissions and coordination with the officials of the Shahid Madani hospital, the researcher attempted sampling. After explaining the research objectives to the subjects, they were asked to sign the informed consent form. Additionally, permission for exercise was obtained from the cardiologist in order to ensure that patients are at ease with exercise. All the rendered services to the subjects were free, and the patients' information was maintained confidential (2029).

At the end of the executive phase of the research, the collected data were analyzed with SPSS software, version 19. After verifying the normal distribution of the data, using the Shapiro-Wilk exploratory test and homogeneity of variances by means of the Leven test, for intra- and inter-group comparisons, the Student's t-test was used in dependent and independent groups (The application of each test is given in the Result Section). A $P<0.05$ was considered as the level of significance.

\section{Results}

During the mentioned period, 31 patients were identified of who, 10 cases were excluded due to the lack of eligibility. Twenty-one patients were enrolled in the study, and 11 patients completed the exercise intervention after random allocation. The participants regularly participated in the designated exercise program and the sample dropouts were 0 (Figure 1).

The mean age \pm standard deviation (SD) of the participants in the training and control groups was 31.29 \pm 12.10 and $30.29 \pm 12.10$ years, respectively, and there was no significant difference between the two groups $(P$ $=0.510)$. In addition, the mean height $\pm \mathrm{SD}$, mean weight $\pm \mathrm{SD}$, and mean $\mathrm{BMI} \pm \mathrm{SD}$ of participants were 171.55 $\pm 15.39 \mathrm{~cm}, 78.80 \pm 6.50 \mathrm{~kg}$, and $25.35 \pm 2.40 \mathrm{~kg} / \mathrm{m}^{2}$, respectively. The comparison of the results between the two groups represented no significant differences in terms of demographic characteristics (Table 1).

Based on the results of the Student's t-test, the effect of the aerobic exercise program on body weight loss $(P$ $=0.011)$, BMI $(P=0.004)$, and body fat percentage $(P$ $=0.001)$ was significant in the training group. Further, the serum $(P=0.002)$ and urine $(P=0.001)$ levels of 8 -hydroxy-2-deoxyguanosine decreased significantly at the end of the intervention compared to baseline in the intervention group. Based on the data in Table 2, intragroup comparisons demonstrated changes in the mean weight and serum 8-hydroxy-2-deoxyguanosine levels between the study groups (Table 2).

\section{Discussion}

The current study aimed to investigate the effect of interval training on oxidative stress indices among

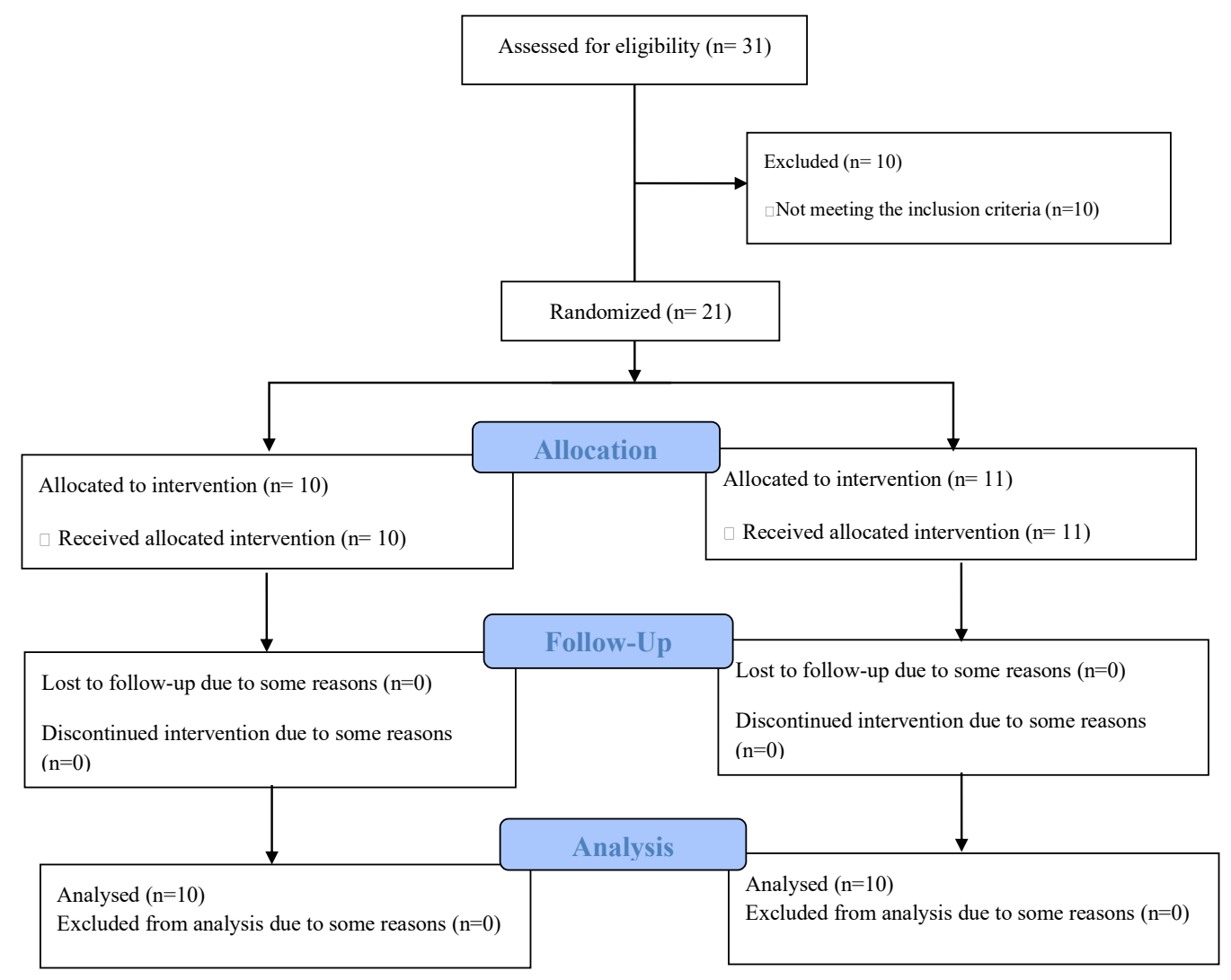

Figure 1. The Flow Chart of the Recruitment and Retention of Participants. 
Table 1. Demographic Characteristics of Patients

\begin{tabular}{lcccc}
\hline \multirow{2}{*}{ Group } & \multicolumn{4}{c}{ Mean \pm SD } \\
\cline { 2 - 5 } & Age (year) & BMI $\left(\mathrm{kg} / \mathrm{m}^{2}\right)$ & Weight $(\mathrm{kg})$ & Height $(\mathrm{cm})$ \\
\hline Training $(\mathrm{n}=11)$ & $31.87 \pm 11.49$ & $24.45 \pm 2.30$ & $77.75 \pm 5.90$ & $168.15 \pm 14.45$ \\
Control $(\mathrm{n}=10)$ & $30.35 \pm 12.10$ & $26.25 \pm 2.50$ & $79.85 \pm 6.11$ & $173.10 \pm 15.80$ \\
$P$ value & 0.402 & 0.703 & 0.512 & 0.369 \\
\hline
\end{tabular}

women in preterm labor undergoing CABG. In recent years, many studies have focused on the reduction of cardiovascular disease and its predisposing factors. In the latest research, oxidative stress and its indices as causative and exacerbating factors for cardiovascular disease are considered more than the other parameters, and ways to improve these indices such as exercise are investigated as well (30). The present study evaluated the effect of eight-week aerobic training on patients who underwent $\mathrm{CABG}$, and a significant change was observed in the (BMI, body fat percentage, and oxidative stress indices in the posttest compared to those of the pretest. Considering that the heart muscle, which is rich in mitochondria, is an important source of ROS production, it is more at risk compared to other body organs (31).

The results of the current study showed that training reduced body fat percentage and BMI, which is consistent with the results of similar studies (32-35). Increasing fat oxidation capability by increasing the level of $\beta$-oxidation enzymes and Krebs cycle productivity following sports activities seem to be the causes of fat burning and a decrease in body fat percentages as well as BMI. More precisely, these indices decrease following doing exercise. On the other hand, decreasing fat percentage and BMI can somehow guarantee the physical health of patients after $\mathrm{CABG}$ and accelerate the treatment process.

8-hydroxy-2-deoxyguanosine is one of the hallmarks of DNA oxidation and has received much attention in recent studies. The researchers believe that regular, not too heavy exercise for this group of people is a healthy and natural way to prevent oxidation and that, the level of 8-hydroxy- 2-deoxyguanosine rises significantly after the exercise. In this regard, Samia et al confirmed this issue (14) and their results are in line with those of the present study.

Moreover, the findings of this study indicated significant changes in the serum and urine levels of 8-hydroxy2-deoxyguanosine after exercise. In this regard, which corroborates with the results of some other studies (14, 36-38). However, it can be argued that aerobic exercise usually increases metabolism while it reduces excessive oxidation and deterioration of the patient's health status through intracellular mechanisms. On the other hand, training with increasing oxygen consumption in the heart tissue may reduce the risk of oxidative stress and its indices in patients with cardiovascular disease.

Limitations

The limitations of the present study were the lack of considering the patients' diet and the small sample size. Therefore, further studies are recommended based on the limitation of the present study.

\section{Conclusions}

In general, aerobic exercise after $\mathrm{CABG}$ reduces fat percentage and BMI, as well as oxidative stress indices. In other words, these exercises decrease oxidative stress indices by increasing tissue metabolism and oxygen consumption. Therefore, the authors suggest that aerobic exercise be added to the rehabilitation programs of patients who underwent cardiac surgeries.

\section{Authors' Contribution}

$\mathrm{KH}$ managed the intervention and participated in data collection. MD contributed to data analysis. MKG Participated in article submission, article preparation, assistance with intervention, and study design.

\section{Conflict of Interests}

Authors declare that they have no conflict of interests.

Table 2. Comparison of Intragroup and Intergroup Changes in Two Groups After Exercise

\begin{tabular}{|c|c|c|c|c|c|}
\hline \multirow{2}{*}{ Variable } & \multirow{2}{*}{ Group } & \multicolumn{2}{|c|}{ Mean \pm SD } & \multicolumn{2}{|c|}{$P$ Value } \\
\hline & & Pretest & Posttest & Intragroup & Intergroup \\
\hline \multirow{2}{*}{ Weight (kg) } & Training $(n=11)$ & $77.75 \pm 5.90$ & $74.03 \pm 5.20$ & 0.011 & \multirow{2}{*}{0.005} \\
\hline & Control $(n=10)$ & $79.85 \pm 6.11$ & $80.15 \pm 6.45$ & 0.312 & \\
\hline \multirow{2}{*}{ BMI $\left(\mathrm{kg} / \mathrm{m}^{2}\right)$} & Training $(n=11)$ & $24.45 \pm 2.30$ & $21.55 \pm 1.71$ & 0.004 & \multirow{2}{*}{0.004} \\
\hline & Control $(n=10)$ & $26.25 \pm 2.50$ & $27.30 \pm 2.60$ & 0.219 & \\
\hline \multirow{2}{*}{ Body fat percentage (\%) } & Training $(n=11)$ & $41.55 \pm 4.80$ & $36.80 \pm 3.95$ & 0.001 & \multirow{2}{*}{0.001} \\
\hline & Control $(n=10)$ & $42.05 \pm 4.91$ & $41.25 \pm 3.99$ & 0.396 & \\
\hline \multirow{2}{*}{$\begin{array}{l}\text { Serum 8-hydroxy-2- } \\
\text { deoxyguanosine }\end{array}$} & Training $(n=11)$ & $341.25 \pm 20.80$ & $250.25 \pm 18.80$ & 0.002 & \multirow{2}{*}{0.002} \\
\hline & Control $(n=10)$ & $345.29 \pm 19.15$ & $340.30 \pm 20.25$ & 0.512 & \\
\hline \multirow{2}{*}{$\begin{array}{l}\text { Urine 8-hydroxy-2- } \\
\text { deoxyguanosine }\end{array}$} & Training $(n=11)$ & $10.40 \pm 2.25$ & $6.55 \pm 2.30$ & 0.001 & \multirow{2}{*}{0.001} \\
\hline & Control $(n=10)$ & $11.29 \pm 2.50$ & $11.90 \pm 2.95$ & 0.275 & \\
\hline
\end{tabular}

Note. SD: Standard deviation; BMI: Body mass index. 


\section{Ethical Issues}

The research project was approved by the Ethics Committee of Tabriz University of Medical Sciences (ethics no. IR.TBZMED.REC.1397.1059; RCT identifier: IRCT20190325043107N2).

\section{Financial Support}

Vice-chancellor of Tabriz University of Medical Sciences supported the study.

\section{Acknowledgments}

The present paper is part of the research project approved by the Clinical Research Development Unit, Shohada Hospital of Tabriz University of Medical Sciences with the ethics code of IR.TBZMED.REC.1397.1059. The researchers would like to give their gratitude to the Research Center and the Health Vice-chancellor of Tabriz University of Medical Sciences for providing financial support in the study.

\section{References}

1. Strzyżewski KW, Pioruńska-Stolzmann M, Majewski W, Kasprzak M, Strzyżewski W. Effect of surgical treatment on lipid peroxidation parameters and antioxidant status in the serum of patients with peripheral arterial disease. Dis Markers. 2013;35(6):647-652. doi:10.1155/2013/530946

2. Engin $\mathrm{KN}$, Yemişci B, Yiğit U, Ağaçhan A, Coşkun C. Variability of serum oxidative stress biomarkers relative to biochemical data and clinical parameters of glaucoma patients. Mol Vis. 2010;16:1260-1271.

3. Dayem AA, Choi HY, Kim JH, Cho SG. Role of oxidative stress in stem, cancer, and cancer stem cells. Cancers (Basel). 2010;2(2):859-884. doi:10.3390/cancers2020859

4. Furukawa S, Fujita T, Shimabukuro M, et al. Increased oxidative stress in obesity and its impact on metabolic syndrome. J Clin Invest. 2004;114(12):1752-1761. doi: $10.1172 /$ jci2 21625

5. Shokoohi M, Olad Saheb Madarek E, Khaki A, Shoorei H, Khaki AA, Soltani M, et al. Investigating the effects of onion juice on male fertility factors and pregnancy rate after testicular torsion/ detorsion by intrauterine insemination method. Int J Womens Health Reprod Sci. 2018;6(4):499505. doi:10.15296/ijwhr.2018.82

6. Abtahi-Eivari SH, Moghimian M, Soltani M, Shoorei H, Asghari R, Hajizadeh H, et al. The effect of Galega officinalis on hormonal and metabolic profile in a rat model of polycystic ovary syndrome. Int J Womens Health Reprod Sci. 2018;6(3):276-282. doi:10.15296/ijwhr.2018.46

7. Sosa V, Moliné T, Somoza R, Paciucci R, Kondoh H, ME LL. Oxidative stress and cancer: an overview. Ageing Res Rev. 2013;12(1):376-390. doi:10.1016/j.arr.2012.10.004

8. Ferguson KK, McElrath TF, Chen YH, Loch-Caruso R, Mukherjee B, Meeker JD. Repeated measures of urinary oxidative stress biomarkers during pregnancy and preterm birth. Am J Obstet Gynecol. 2015;212(2):208.e201-208. doi:10.1016/j.ajog.2014.08.007

9. Sultana Z, Maiti K, Aitken J, Morris J, Dedman L, Smith R. Oxidative stress, placental ageing-related pathologies and adverse pregnancy outcomes. Am J Reprod Immunol.
2017;77(5). doi:10.1111/aji.12653

10. Young IS, Woodside JV. Antioxidants in health and disease. J Clin Pathol. 2001;54(3):176-186. doi:10.1136/jcp.54.3.176

11. Chehreh R, Karamolahi Z, Aevazi A, Borji M, Saffar A. Prevalence of preterm birth recurrence and related factors in Ilam. Iran J Obstet Gynecol Infertil. 2018;21(10):20-29. doi:10.22038/ijogi.2018.12241

12. Wu LL, Chiou CC, Chang PY, Wu JT. Urinary 8-OHdG: a marker of oxidative stress to DNA and a risk factor for cancer, atherosclerosis and diabetics. Clin Chim Acta. 2004;339(1-2):1-9. doi:10.1016/j.cccn.2003.09.010.

13. de Pinho RA, de Araújo MC, de Melo Ghisi GL, Benetti M. [Coronary heart disease, physical exercise and oxidative stress]. Arq Bras Cardiol. 2010;94(4):549-555. doi:10.1590/ s0066-782x2010000400018

14. Samia BA, Youssef GA. Changes in urinary 8-hydroxydeoxyguanosine levels during heptathlon race in professional female athletes. J Hum Kinet. 2014;41:107-111. doi:10.2478/hukin-2014-0038

15. Nojima $\mathrm{H}$, Watanabe $\mathrm{H}$, Yamane $\mathrm{K}$, et al. Effect of aerobic exercise training on oxidative stress in patients with type 2 diabetes mellitus. Metabolism. 2008;57(2):170-176. doi:10.1016/j.metabol.2007.08.021

16. Friedenreich CM, Pialoux V, Wang Q, et al. Effects of exercise on markers of oxidative stress: an ancillary analysis of the Alberta Physical Activity and Breast Cancer Prevention Trial. BMJ Open Sport Exerc Med. 2016;2(1):e000171. doi:10.1136/bmjsem-2016-000171

17. Rodríguez-Pascual F, Busnadiego O, Lagares D, Lamas S. Role of endothelin in the cardiovascular system. Pharmacol Res. 2011;63(6):463-472. doi:10.1016/j.phrs.2011.01.014

18. Abdolmaleki Z, Saleh Sedghpour B, Bahram A, Abdolmaleki F. Validity and reliability of the physical self-description questionnaire among adolescent girls. J Appl Psychol. 2011;4(16):42-55. [Persian].

19. Hejazi K, Ghahremani Moghaddam M, Darzabi T. Effects of an 8-week aerobic exercise program on some indicators of oxidative stress in elderly women. Salmand: Iranian Journal of Ageing. 2019;13(4):506-517. doi:10.32598/sija.13.4.506

20. Zomorrodi A, Mohammadipoor Anvari H, Kakaei F, Solymanzadeh F, Khanlari E, Bagheri A. Bolus injection versus infusion of furosemide in kidney transplantation: a randomized clinical trial. Urol J. 2017;14(2):3013-3017. doi:10.22037/uj.v14i2.3787

21. Aghamohammadi D, Gargari RM, Fakhari S, Bilehjani E, Poorsadegh S. Classic versus Inguinal approach for obturator nerve block in transurethral resection of bladder cancer under spinal anesthesia: a randomized controlled trial. Iranian Journal of Medical Sciences. 2018;43(1):75-80.

22. Bakhshaei MH, Manuchehrian N, Khoshraftar E, Mohamadipour Anvary H, Sanatkarfar M. Analgesic effects of intrathecal sufentanil added to lidocaine 5\% in elective cesarean section. Acta Med Iran. 2010;48(6):380-384.

23. Sadeghi-Bazargani H, Samadirad B, Moslemi F. A decade of road traffic fatalities among the elderly in north-West Iran. BMC Public Health. 2018;18(1):111. doi:10.1186/s12889017-4976-2

24. Darghahi R, Mobaraki-Asl N, Ghavami Z, Pourfarzi F, Hosseini-Asl S, Jalilvand F. Effect of cell-free fetal DNA on spontaneous preterm labor. J Adv Pharm Technol Res. 2019;10(3):117. doi: 10.4103/japtr.JAPTR_371_18. 
25. Mallah F, Tasbihi P, Navali N, Azadi A. Urinary incontinence during pregnancy and postpartum incidence, severity and risk factors in Alzahra and Taleqani hospitals in Tabriz, Iran, 2011-2012. Int J Womens Health Reprod Sci. 2014;2(3):178-185. doi: 10.15296/ijwhr.2014.26.

26. Mallah F, Nazari F, Navali N, Hajipour B. Comparison of direct visual inspection (DVI) with pap smear in diagnosis of precancerous lesion of cervix. Life Sci J. 2012;9(4):25562560.

27. Bastani P, Shoari N, Haj Ebrahimi S, Mallah F, Azadi A. Comparison of performing and not-performing the prophylactic surgery for urinary incontinence in advanced pelvic organ prolapse. Int J Womens Health Reprod Sci. 2014;2(5):311-315. doi:10.15296/ijwhr.2014.51

28. Ziyadi S, Bastani P, Homayouni A, Mohammad-AlizadehCharandabi S, Mallah F. Probiotics and usage in urinary tract infection. In: Watson RR, Preedy VR, eds. Probiotics, Prebiotics, and Synbiotics: Bioactive Foods in Health Promotion. London: Elsevier; 2016:827-830. doi:10.1016/ b978-0-12-802189-7.00063-0

29. Navali N, Azhary Shokoufe L, Mallah F, Bastani P, Mashrabi O. Comparing therapeutic effects of Metformin and Pioglitazone in Polycystic ovary syndrome (PCOS). Pak J Med Sci. 2012;28(3):390-394.

30. Atadokht A, Hashemi J, Habibi Y, Fallahi V, Rahimi F. The role of illness perception and perceived social support in predicting psychological distress in patients with multiple sclerosis. Nursing and Midwifery Journal. 2018;15(11):863873. [Persian].

31. Shakir DK, Rasul KI. Chemotherapy induced cardiomyopathy: pathogenesis, monitoring and management. J Clin Med Res. 2009;1(1):8-12. doi:10.4021/ jocmr2009.02.1225
32. Thomas GA, Cartmel B, Harrigan M, et al. The effect of exercise on body composition and bone mineral density in breast cancer survivors taking aromatase inhibitors. Obesity (Silver Spring). 2017;25(2):346-351. doi:10.1002/ oby. 21729

33. Rock CL, Doyle C, Demark-Wahnefried W, et al. Nutrition and physical activity guidelines for cancer survivors. CA Cancer J Clin. 2012;62(4):243-274. doi:10.3322/caac.21142

34. Schwingshackl L, Dias S, Strasser B, Hoffmann G. Impact of different training modalities on anthropometric and metabolic characteristics in overweight/obese subjects: a systematic review and network meta-analysis. PLoS One. 2013;8(12):e82853. doi:10.1371/journal.pone.0082853

35. Giangregorio LM, McGill S, Wark JD, et al. Too fit to fracture: outcomes of a Delphi consensus process on physical activity and exercise recommendations for adults with osteoporosis with or without vertebral fractures. Osteoporos Int. 2015;26(3):891-910. doi:10.1007/s00198014-2881-4

36. Rahimi R, Sharafi H. The effect of a bout of resistance exercise on 8-Hydroxy-2'-Deoxyguanosine in athletes and non-athletes. Knowledge and Health. 2012;7(1):1-7. [Persian].

37. Shin YA, Lee JH, Song W, Jun TW. Exercise training improves the antioxidant enzyme activity with no changes of telomere length. Mech Ageing Dev. 2008;129(5):254-260. doi:10.1016/j.mad.2008.01.001

38. Pourfazeli B, Azamian Jazi A, Faramarzi M, Mortazavi MJ. Effect of regular aerobic training on oxidative damage markers of lipids and proteins in rats exposed to radiation emitted by the Wi-Fi router. Journal of Torbat Heydariyeh University of Medical Sciences. 2017;5(2):11-19. [Persian].

(C) 2020 The Author(s); This is an open-access article distributed under the terms of the Creative Commons Attribution License (http:// creativecommons.org/licenses/by/4.0), which permits unrestricted use, distribution, and reproduction in any medium, provided the original work is properly cited. 